\title{
Missing the Mark: Inaccuracy of administrative data in identification of hospitalized patients with pneumonia and results of a systematic clinical reclassification process on readmission rates
}

\author{
Marcus D. Ruopp*1,2, Joel C. Boggan ${ }^{2,3}$, Thomas L. Holland ${ }^{2,4,5}$, Mary Jane Stillwagon ${ }^{6}$, Joseph A. Govert ${ }^{7}$, Jonathan \\ G. $\mathrm{Bae}^{2,4,8}$ \\ ${ }^{1}$ Geriatrics and Extended Care Service, Veterans Affairs Boston Healthcare System, Boston, MA, United States \\ ${ }^{2}$ Division of General Internal Medicine, Department of Medicine, Duke University Medical Center, Durham, NC, United States \\ ${ }^{3}$ Hospital Medicine, Department of Medicine, Durham Veterans Affairs Medical Center, Durham, NC, United States \\ ${ }^{4}$ Hospital Medicine, Duke University Medical Center, Durham, NC, United States \\ ${ }^{5}$ Division of Infectious Diseases, Department of Medicine, Duke University Medical Center, Durham, NC, United States \\ ${ }^{6}$ Department of Nursing, Duke University Health System, Durham, NC, United States \\ ${ }^{7}$ Division of Pulmonary, Allergy, and Critical Care Medicine, Department of Medicine, Duke University Medical Center, Durham, \\ NC, United States \\ ${ }^{8}$ Patient Safety and Clinical Quality, Duke University Health System, Durham, NC, United States
}

Received: September 21, 2017

DOI: $10.5430 /$ jha.v6n6p35
Accepted: November 12, $2017 \quad$ Online Published: November 20, 2017

URL: https://doi.org/10.5430/jha.v6n6p35

\begin{abstract}
Objective: Pneumonia readmissions carry financial ramifications under the Hospital Readmissions Reduction Program (HRRP). As readmission determination utilizes administrative data, healthcare systems should evaluate accuracy of pneumonia diagnoses. We sought to develop a systemic process for pneumonia classification review and determine potential effects on pneumonia readmissions in a tertiary academic medical center in the United States.

Methods: We performed independent reviews of all pneumonia discharges within 48 hours of discharge over a one-year period. We reclassified all pneumonia discharges into four categories based on the Centers for Disease Control and Prevention reference standard. Secondary review of discordant classifications was performed by discharging providers to determine final diagnosis. The primary outcome was readmission rate within 30 days by pneumonia clinical classification category.

Results: Two hundred seventy-eight discharges were reviewed, with overall readmission rate of $18.0 \%$. Independent review confirmed 191 cases (68.7\%) as definite or probable pneumonia, while 87 cases (31.3\%) were classified as either probably not or not pneumonia. Readmission rates differed significantly between cases reviewed as pneumonia vs. those reviewed as unlikely to be pneumonia $(14.1 \%$ vs. $26.4 \%, p<.02)$. Discharging attending physicians agreed with independent reviewers in $58 / 87$ cases $(66.6 \%$ ), attenuating readmission differences (rate $16.8 \%$ for those finalized as pneumonia vs. $22.4 \%$ for another diagnosis, $p=.32$ ). Pneumonia readmissions were reduced by $1.2 \%$ using the classification standard.

Conclusions: Complex conditions such as pneumonia may be inaccurately diagnosed in many patients, potentially affecting penalties associated with readmission rates. Therefore, it is imperative that healthcare systems adopt systematic review processes to standardize diagnoses and improve comparative administrative data.
\end{abstract}

Key Words: Pneumonia, Readmissions, Clinical documentation improvement, Quality improvement, Hospital medicine

*Correspondence: Marcus D. Ruopp; Email: Marcus.ruopp@va.gov; Address: Veterans Affairs Boston Healthcare System, 940 Belmont Street, Brockton, MA, 02301, United States. 


\section{INTRODUCTION}

Hospital readmissions are increasingly scrutinized as a metric for health care quality and improved patient care, prompting initiation of the Centers for Medicare \& Medicaid Services (CMS) Hospital Readmissions Reduction Program (HRRP) in October 2012. ${ }^{[1,2]}$ The HRRP initially required CMS to reduce payments for three conditions: pneumonia, congestive heart failure, and acute myocardial infarction, and was expanded in 2015 to include acute exacerbation of chronic obstructive pulmonary disease and elective total hip and knee arthroplasty. ${ }^{[3,4]}$ A hospital's excess readmission ratio is a risk-adjusted measure of a hospital's readmission performance based on patient demographics, comorbidities, and frailty, compared to the national average for similar patients with that applicable condition. Penalties under HRRP have been widespread, with payments reduced to $78 \%$ of hospitals in 2016 and penalties projected to total $\$ 420$ million. ${ }^{[5]}$ The effect and financial ramifications of the HRRP make it imperative that hospitals and healthcare systems evaluate their methodology to capture accurate data in the calculation of readmission rates.

The backbone of the CMS analysis is driven by administrative/claims data, which numerous studies have used to evaluate the current readmission environment. ${ }^{[6-8]}$ Multiple studies have documented the discordance between claims and clinical data in the diagnosis of myocardial infarction and pneumonia. ${ }^{[9-11]}$ For example, one study estimated the sensitivity of five different claims-based pneumonia definitions against a clinical reference standard ranging from only $48 \%$ to $66 \% .{ }^{[1]}$ In an additional cohort of 7,615 intensive care unit admissions, investigators found significant overestimation and misclassification of pneumonia as the hospital discharge diagnosis in intensive care unit patients. ${ }^{[12]}$

The accuracy of pneumonia claims data is complicated further by classifications of patients with different severities of pneumonia into diagnostic categories such as sepsis and respiratory failure. Based on simulation methods, many hospitals could improve pneumonia readmission rates by reclassifying pneumonia patients as having either sepsis or respiratory failure, depending on clinical parameters. ${ }^{[13]}$ In addition, there is wide variation in hospitals' use of principal diagnoses of sepsis or respiratory failure in patients with pneumonia. ${ }^{[14]}$ Between 2003 to 2009, one study discovered hospitalization and inpatient mortality rates for patients with a principal diagnosis of pneumonia decreased substantially. However, when the principal diagnoses of sepsis or respiratory failure with a secondary diagnosis of pneumonia were included, the declines in the hospitalization rate and inpatient mortality were attenuated; ${ }^{[15]}$ this suggests an association of these results with temporal trends in diagnostic coding. In or- der for comparisons of administrative data to be accurate and meaningful, hospitals need defined systems processes that can accurately reflect final diagnoses and illness severities.

We sought to develop a systematic process for evaluating pneumonia classification within administrative data to determine potential effects on publicly reported data. Specifically, we reviewed whether billing data used in calculating readmission rates at our institution accurately classified pneumonia diagnoses as compared to a clinical reference standard. Additionally, in cases diverging from the clinical reference standard, we examined the effects of data reclassification of pneumonia discharges on 30-day readmission rates.

\section{Methods}

Duke University Hospital is a tertiary academic medical center with approximately 38,000 discharges each year. All cases of pneumonia were identified using CMS definitions and endorsed by the National Quality Forum for patients admitted between December 1, 2012 and December 31, 2013. ${ }^{[16]}$ Patients were excluded if they were $<18$ years of age, died, were discharged against medical advice, or were an index admission transferred to another acute facility.

A multidisciplinary Pneumonia Readmission Review Team was formed by members of the divisions of Hospital Medicine, Infectious Diseases, and Pulmonary Medicine, as well as Hospital Leadership, to review all identified discharges with a preliminary primary diagnosis of pneumonia (International Classification of Diseases, Ninth Revision, Clinical Modification diagnosis codes: 480.X, 481, 482.XX, 483.X, 485, 486, 487.0). ${ }^{[17]}$ Case review included review of full admission and hospitalization progress, consultation, and discharge documentation, as well as laboratory data and radiographs to validate the diagnosis based on the Centers for Disease Control and Prevention algorithm for defining clinical pneumonia.

Based on case review, one of three reviewers designated the "true" primary clinical diagnosis into one of the following four categories:

(1) Definite Pneumonia: Meets clinical reference standard criteria.

(2) Probable Pneumonia: Does not meet clinical reference standard criteria, but no alternative diagnosis is identified or likely.

(3) Probably Not Pneumonia: Does not meet clinical reference standard criteria with a possible alternative diagnosis.

(4) Not Pneumonia: Does not meet the clinical reference standard criteria and there is an alternative diagnosis.

If the independent reviewer determined the index case fell 
within categories 1 or 2 , then the case was deemed to be pneumonia and a preliminary diagnosis was confirmed. If the independent reviewer determined the case fell within categories 3 or 4 , then the case was sent back to the discharging provider for review via secure email. This email was accompanied by a brief explanation of the quality improvement effort, the review process utilizing the Centers for Disease Control and Prevention algorithm, and a possible/probable alternative diagnosis. Upon further review, if the discharging provider determined the index case to be classified as either definite or probable pneumonia, then the index case remained categorized as pneumonia and the preliminary diagnosis was confirmed. However, if upon review, the discharging provider agreed the case was not pneumonia, then the diagnosis was coded based on amended documentation. The process design included goals for the completion of the initial review within 48 hours and completion of the secondary process and documentation changes within 7 business days.

Table 1. Patient and hospitalization characteristics by pneumonia status after independent review

\begin{tabular}{|c|c|c|c|c|}
\hline & All Subjects & Not Pneumonia & Pneumonia & $p$-value \\
\hline All encounters, no. (\%) & 278 & $87(31.29)$ & $191(68.71)$ & \\
\hline \multicolumn{5}{|l|}{ Pneumonia score, no. (\%) } \\
\hline - 1 & $94(33.8)$ & & $94(49.2)$ & \\
\hline - 2 & $97(34.9)$ & & $97(50.8)$ & \\
\hline - 3 & $64(23.0)$ & $64(73.6)$ & & \\
\hline - 4 & $23(8.3)$ & $23(26.4)$ & & \\
\hline Age (years) & & & & .36 \\
\hline - Mean $(S D)$ & $64.1(17.5)$ & $62.7(19.2)$ & $64.8(16.7)$ & \\
\hline - Median (IQR) & $67.0(54.0-77.0)$ & $67.0(48.0-77.0)$ & $67.0(55.0-76.0)$ & \\
\hline Gender, no. $(\%)$, & & & & .85 \\
\hline - Female & $127(45.7)$ & $39(44.8)$ & $88(46.1)$ & \\
\hline - Race, no. (\%) & & & & .37 \\
\hline American Indian & $13(4.7)$ & $3(3.5)$ & $10(5.2)$ & \\
\hline Black or African American & $84(30.2)$ & $31(35.6)$ & $53(27.8)$ & \\
\hline White or Caucasian & $181(65.1)$ & $53(60.9)$ & $128(67.0)$ & \\
\hline Medicaid or Medicare, no. (\%) & $203(73.0)$ & $68(78.2)$ & $135(70.7)$ & .19 \\
\hline CAD, no. $(\%)$ & $55(19.8)$ & $17(19.5)$ & $38(19.9)$ & .95 \\
\hline Diabetes, no. (\%) & $68(24.5)$ & $20(23.0)$ & $48(25.1)$ & .70 \\
\hline COPD, no. (\%) & $71(25.5)$ & $19(21.8)$ & $52(27.2)$ & .34 \\
\hline Asthma, no. (\%) & $15(5.4)$ & $3(3.5)$ & $12(6.3)$ & .33 \\
\hline Heart failure, no. $(\%)$ & $49(17.6)$ & $10(11.5)$ & $39(20.4)$ & .07 \\
\hline Mental health/substance abuse, no. (\%) & $121(43.5)$ & $45(51.7)$ & $76(39.8)$ & .06 \\
\hline Sickle cell, no. $(\%)$ & $7(2.5)$ & $6(6.9)$ & $1(0.5)$ & $<.01$ \\
\hline Comorbidity types, no. (\%) & & & & .43 \\
\hline - None & $33(11.9)$ & $11(12.6)$ & $22(11.5)$ & \\
\hline - 1 to 3 & $205(73.7)$ & $67(77.0)$ & $138(72.3)$ & \\
\hline - 4 or more & $40(14.4)$ & $9(10.3)$ & $31(16.2)$ & \\
\hline Length of stay in days & & & & .06 \\
\hline - Mean $(S D)$ & $4.9(4.9)$ & $5.7(7.1)$ & $4.5(3.4)$ & \\
\hline - Median (IQR) & $3.0(2.0-6.0)$ & $4.0(2.0-7.0)$ & $3.0(2.0-6.0)$ & \\
\hline - Emergency department w/in 30 days, no. (\%) & $49(17.6)$ & $22(25.3)$ & $27(14.1)$ & .02 \\
\hline Days to readmission $(\leq 30)$ & & & & .95 \\
\hline - Mean $(S D)$ & $13.7(8.4)$ & $13.8(8.6)$ & $13.6(8.4)$ & \\
\hline - Median (IQR) & $11.5(7.0-18.0)$ & $12.0(7.0-18.0)$ & $10.0(7.0-20.0)$ & \\
\hline - Readmission w/in 30 days, no. (column\%, row\%) & $50(18.0)$ & $23(26.4)$ & $27(14.1)$ & .01 \\
\hline
\end{tabular}

Note. CAD: coronary artery disease; IQR: interquartile range; $S D$ : standard deviation

\section{Statistical analysis}

The primary outcome was readmission rate within 30 days by pneumonia clinical classification category. Descriptive statistics were performed by classification status at each stage of the review process. Statistical testing was conducted to measure the association of misclassification and patient characteristics. Categorical data were evaluated with Chi-square testing and continuous data with student's T-tests. All analysis was conducted using SAS version 9.4 (Cary, NC). Due to Published by Sciedu Press retrospective analysis of existing data, the study was granted exempt status by the Duke University Institutional Review Board (Pro00048675).

\section{Results}

Overall, 278 pneumonia discharges were reviewed during the study period. The mean age was 64.1 years, and $203(73 \%)$ had Medicaid or Medicare (see Table 1). The median length of stay was 3.0 days. The overall readmission rate of this 
population was $18.0 \%$ with a median time to readmission of 11.5 days. Independent review found 191 cases (68.7\%) were definite or probable pneumonia (Categories 1 and 2) in concordance with the preliminary discharging diagnosis, while 87 cases $(31.3 \%)$ were determined to be probably not or not pneumonia (Categories 3 and 4). These groups had significantly different readmission rates: for cases independently reviewed and felt unlikely to be pneumonia, the readmission rate was $26.4 \%$, while for those confirmed to be pneumonia, the readmission rate was $14.1 \%(p<.02$, see Table 1$)$.

For the 87 reviewed cases that were felt to be inconsistent with pneumonia after independent review, the primary discharging attending agreed with the independent reviewer in 58 cases $(66.6 \%$, representing $20.9 \%$ of the preliminary pneumonia discharges, see Figure 1). As a result, these 58 cases were ultimately coded under an alternative diagnosis (see Table 2).

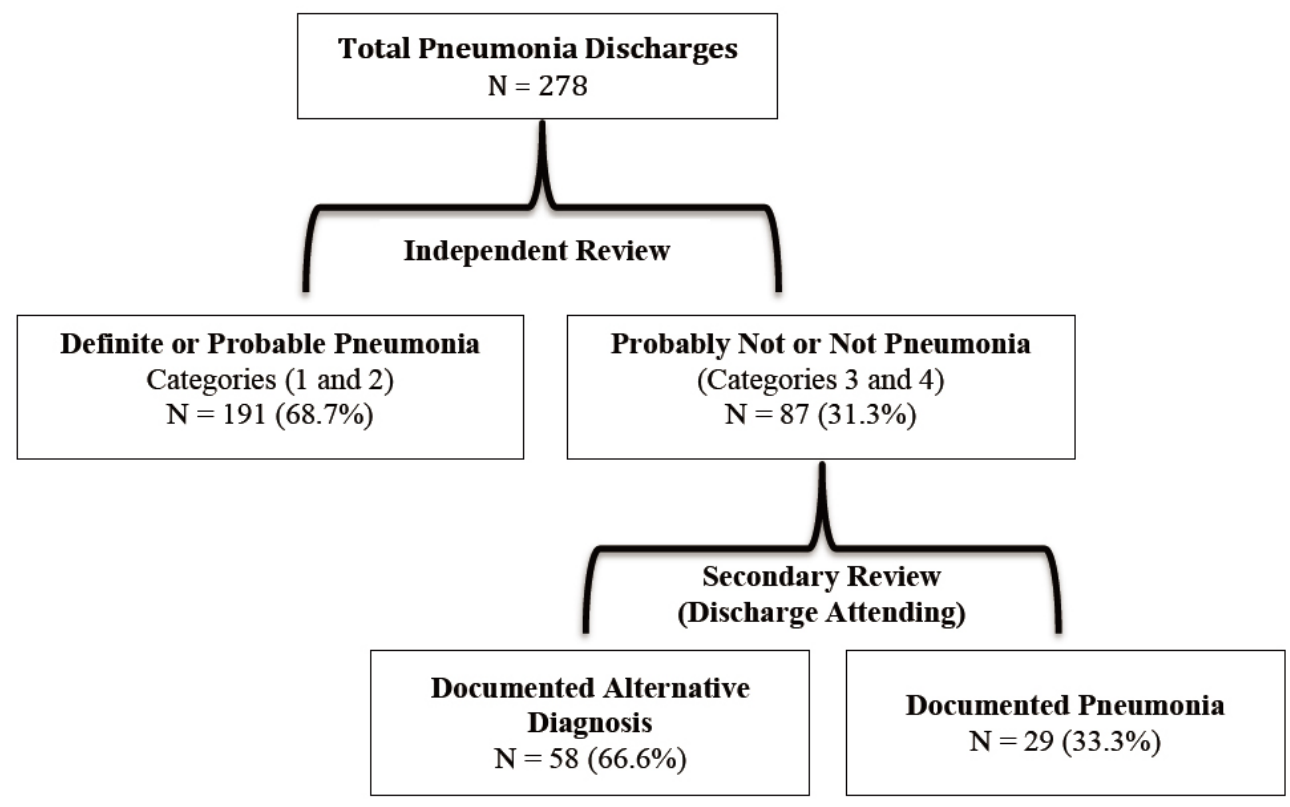

Figure 1. Flowchart of pneumonia process

In the final analysis for the 278 discharges initially diagnosed as pneumonia, 220 were finalized as pneumonia based on either independent reviewer agreement with the diagnosis of pneumonia or primary attending disagreement with the independent reviewer's conclusion. The median length of stay was not significantly different between the final diagnosis of pneumonia group and the alternative diagnosis group (3.0 days vs. 4.0 days, $p=.14$ ). Additionally, the difference in readmission rate did not differ significantly for the groups (22.4\% for the alternative diagnosis group and $16.8 \%$ for the pneumonia group, $p=.32$ ) (see Table 3). On average, it took 7.8 days to receive notification of a pneumonia discharge and 3.4 days for review and amendment of discharge documentation. Overall, the use of the clinical reference standard reduced the rate of pneumonia readmissions from a possible $18.0 \%$ to $16.8 \%$.

\section{Discussion}

The introduction of the HRRP has significant ramifications for hospitals capturing metrics for healthcare quality and subsequent financial impacts. As these metrics and penalties are compiled from administrative data, it is imperative that these claims data are accurate and hospitals adopt system processes to capture accurate data. In this study, our independent reviewers identified that $31.3 \%$ of pneumonia cases discharged from our hospital over a 13-month period were likely not pneumonia, and $20 \%$ were formally coded as an alternative diagnosis based on consultation and re-review of documentation by the primary attending physician. Overall, these diagnostic changes revised our potential readmission rate for pneumonia diagnoses by an absolute $1.2 \%$.

This study reinforces the concern for discordance between claims data and clinical-based reference standards, especially for the diagnosis of pneumonia. ${ }^{[9-11]}$ Pneumonia has multiple clinical manifestations and etiologies, making it difficult to capture using standardized coding schemes. ${ }^{[11,12]}$ In addition, variable training with and use of diagnostic criteria by clinicians for these heterogeneous clinical entities further compounds the difficulties of appropriate labeling for medical coders. 
Table 2. Alternative final diagnoses for patients determined to not have pneumonia after secondary review $(\mathrm{n}=58)$

\begin{tabular}{lll}
\hline & Frequency & Percent Alternative Diagnoses (\%) \\
\hline Disease of the respiratory system (ICD-9 codes: 460-519) & 33 & 56.9 \\
- Pneumonitis & 6 & 10.3 \\
- Influenza & 6 & 10.3 \\
- Obstructive chronic bronchitis with (acute) exacerbation & 4 & 6.9 \\
- Acute bronchitis & 3 & 5.2 \\
- Upper respiratory infection & 3 & 5.2 \\
- Acute chest syndrome & 2 & 3.4 \\
- Bronchiolitis & 2 & 3.4 \\
- Idiopathic pulmonary fibrosis & 1 & 1.7 \\
- Other & 6 & 10.3 \\
Symptoms, signs, and ill-defined conditions (ICD-9 codes: 780-799) & 13 & 22.4 \\
- Dyspnea & 4 & 6.9 \\
- Hemoptysis & 3 & 5.2 \\
- Fever & 3 & 5.2 \\
- Dysphagia & 1 & 1.7 \\
- Hypoxia & 1 & 1.7 \\
- Altered mental status & 1 & 1.7 \\
Endocrine, nutritional and metabolic diseases, and immunity disorders & 2 & 3.4 \\
(ICD-9 codes: $240-279)$ & & \\
- Cystic fibrosis exacerbation & 2 & 3.4 \\
Injury and poisoning (ICD-9 codes: 800-999) & 4 & 6.9 \\
- Sepsis & 4 & 6.9 \\
Neoplasms (ICD-9 codes: 140-239) & 3 & 5.2 \\
- Metastatic breast cancer & 1 & 1.7 \\
- Lung cancer & 1 & 1.7 \\
- Metastatic sarcoma & 1 & 1.7 \\
Infectious and parasitic diseases (ICD-9 codes: $001-139$ ) & 1 & 1.7 \\
- Candidemia & 1 & 1.7 \\
Diseases of the blood and blood-forming organs (ICD-9 codes: 280-289) & 1 & 1.7 \\
- Leukocytosis & 1 & 1.7 \\
Diseases of the circulatory system (ICD-9 codes: $390-495)$ & 1 & 1.7 \\
- Acute on chronic congestive heart failure & 1 & 1.7 \\
\hline
\end{tabular}

Note. ICD-9: International Classification of Diseases, Ninth Revision

Table 3. Hospitalization characteristics of final pneumonia status after secondary review

\begin{tabular}{|c|c|c|c|c|}
\hline & All Subjects & Not Pneumonia & Pneumonia & $p$-value \\
\hline All encounters, no. & 278 & $58(20.9)$ & $220(79.1)$ & \\
\hline \multicolumn{5}{|l|}{ Pneumonia score, no. (\%) } \\
\hline - 1 & $94(33.8)$ & & $94(42.7)$ & \\
\hline - 2 & $97(34.9)$ & & $97(44.1)$ & \\
\hline - 3 & $64(23.0)$ & $36(62.1)$ & $28(12.7)$ & \\
\hline - 4 & $23(8.3)$ & $22(37.9)$ & $1(0.5)$ & \\
\hline Length of inpatient stay in days & & & & .15 \\
\hline - Mean $(S D)$ & $4.9(4.9)$ & $5.7(8.2)$ & $4.6(3.5)$ & \\
\hline - Median (IQR) & $3.0(2.0-6.0)$ & $4.0(2.0-6.0)$ & $3.0(2.0-6.0)$ & \\
\hline Emergency department w/in 30 days, no. (\%) & $49(17.6)$ & $12(20.7)$ & $37(16.8)$ & .49 \\
\hline Days to readmission $(\leq 30)$ & & & & .99 \\
\hline - Mean $(S D)$ & $13.7(8.4)$ & $13.7(9.1)$ & $13.7(8.3)$ & \\
\hline - Median (IQR) & $11.5(7.0-18.0)$ & $15.0(6.0-17.0)$ & $10.0(8.0-18.0)$ & \\
\hline Readmission w/in 30 days, no. (column\%, row\%) & $50(18.0)$ & $13(22.4)$ & $37(16.8)$ & .32 \\
\hline
\end{tabular}

Note. IQR: interquartile range; $S D$ : standard deviation 
This study suggests that accurate reclassification of pneumonia diagnosis may have the potential to affect readmission rates. Cases identified by independent reviewers as likely secondary to a different diagnosis based on the clinical reference standard had an almost two-fold significantly higher readmission rate. Although this result was not statistically significant after secondary review and final classification by primary attendings, a six-point difference in readmission rate remained between the groups with an absolute $1.2 \%$ reduction in readmission rates for pneumonia diagnoses. Overall, our review period was likely too short to capture an adequate volume of pneumonia patients, leaving us underpowered to determine whether this difference is real.

As noted in previous studies, such large changes may affect whether or not a hospital is penalized for readmissions. ${ }^{[13,18]}$ National focus and penalties based on readmissions for specific conditions are having an important effect on the healthcare system as a whole, as overall and specific condition readmissions have been decreasing over time. ${ }^{[18-20]}$ Yet with increasing penalties looming and studies showing diagnostic heterogeneity across different health systems, hospitals may feel incentivized to prioritize improving documentation and administrative data collection over improving clinical care.

Creating a systematic review process of pneumonia diagnoses with a clinical standard has the potential to lead to more uniform diagnoses and more accurate administrative data for comparisons. Although such review increases the complexity of diagnosis and coding processes, it may be necessary to ensure accuracy. Our study demonstrates that independent review alone may alter many pneumonia diagnosis codes, although only two-thirds of suggested changes were adopted by the primary discharging provider, which supports the argument that a review process should include both an independent reviewer and the primary attending provider.

This study demonstrates claims data verifications can be performed in a timely manner, with only 11.2 days from discharge needed for review and amendment of discharge documentation. For systems focusing on both accuracy and efficiency, a similar process with independent review and subsequent primary verification could be performed in time to allow for billing. Additionally, such a short verification timeframe still would allow for relatively rapid data feedback for ongoing clinical care improvement projects.

Our study must be considered in light of some limitations. First, the review performed after discharge was retrospective (rather than real-time). Some have suggested that present on admission diagnoses could be used instead of discharge diagnoses for the HRRP — a suggestion we support for purposes of eliminating the retrospective nature of the review, thereby allowing for real-time review. However, it has been suggested that present on admission diagnoses also vary between hospitals and could exclude sicker patients with pneumonia that are labeled under the diagnosis of sepsis or respiratory failure. ${ }^{[21]}$ Second, diagnosis codes for sepsis and respiratory failure were not evaluated to determine if they met a clinical reference standard for pneumonia, so future work should be targeted at these diagnosis codes. Third, the cases captured and evaluated were those documented only as pneumonia on discharge; consequently, there was likely a subset of missed patients who were not accurately documented as pneumonia on discharge. Fourth, there are numerous diagnoses with the potential to be misclassified as (or as not) pneumonia diagnoses, as well as other diagnoses for which there is increased payor scrutiny, so one challenge at the health system level is identifying which diagnoses health systems should prioritize for review. Finally, due to resource restraints, only one independent reviewer evaluated each case, so we were not able to assess reliability between reviewers. Although two independent reviewers with a third independent arbitrator may have been optimal from a study standpoint, such a review process would be more difficult to implement and sustain.

In conclusion, complex conditions such as pneumonia may be inaccurately diagnosed in many patients, which presents significant difficulties for accurate data aggregation and quality measurement. The effect of such heterogeneity in clinical classification has the potential to affect core hospital statistics, such as readmission rates. Therefore, it is crucial for hospitals and healthcare organizations to institute systematic processes to create and gather accurate data, and it is essential for insurers and quality observers to continuously validate data streams.

\section{CONFLiCTS OF INTEREST Disclosure}

The authors declare they have no conflicts of interest.

\section{Disclosures}

Thomas Holland: Consultant for Basilea Pharmaceutica (ceftobiprole), Genentech (immunotherapeutic), Motif Bio (iclaprim), The Medicines Company (oritavancin) and Theravance (telavancin); scientific advisory board for Motif Bio (iclaprim); royalties from UpToDate.

Joel Boggan: Consultant for SV Analytics. 


\section{REFERENCES}

[1] Goldfield N, McCullough E, Hughes JS, et al. Identifying potentially preventable readmissions. Health Care Financ Rev. 2008; 30: 75-91. PMid: 19040175.

[2] Jencks SF, Williams MV, Coleman EA. Rehospitalizations among patients in the Medicare fee-for-service program. N Engl J Med. 2009; 360: 1418-1428. PMid: 19339721. https://doi.org/10.1056/ NEJMsa0803563

[3] McIlvennan CK, Eapen ZJ, Allen LA. Hospital readmissions reduction program. Circulation. 2015; 131: 1796-1803. PMid: 25986448. https://doi.org/10.1161/CIRCULATIONAHA.114.010270

[4] Centers for Medicare \& Medicaid Services. Readmissions Reduction Program (HRRP). Accessed October 25, 2016. Available from: https://www.cms.gov/medicare/medicare-fee-for-ser vice-payment/acuteinpatientpps/readmissions-reduc tion-program.html

[5] Medicare Payment Advisory Commission. Report to the Congress: Medicare Payment Policy. 2016. Available from: http://www.medpac.gov/docs/default-source/reports/ march-2016-report-to-the-congress-medicare-payment - policy.pdf?sf vrsn=0

[6] Dharmarajan K, Hsieh AF, Lin Z, et al. Diagnoses and timing of 30-day readmissions after hospitalization for heart failure, acute myocardial infarction, or pneumonia. JAMA. 2013; 309: 355-363. PMid: 23340637. https://doi.org/10.1001/jama.2012.216476

[7] Nuti SV, Qin L, Rumsfeld JS, et al. Association of admission to Veterans Affairs hospitals vs non-Veterans Affairs hospitals with mortality and readmission rates among older men hospitalized with acute myocardial infarction, heart failure, or pneumonia. JAMA. 2016; 315 : 582-592. PMid: 26864412. https://doi.org/10.1001/jama. 2 016.0278

[8] Lindenauer PK, Bernheim SM, Grady JN, et al. The performance of US hospitals as reflected in risk-standardized 30-day mortality and readmission rates for Medicare beneficiaries with pneumonia. J Hosp Med. 2010; 5: E12-18. PMid: 20665626. https: //doi.org/10.1002/jhm. 822

[9] Jollis JG, Ancukiewicz M, DeLong ER, et al. Discordance of databases designed for claims payment versus clinical information systems. Implications for outcomes research. Ann Intern Med. 1993; 119: 844-815. PMid: 8018127. https://doi.org/10.7326/00 03-4819-119-8-199310150-00011

[10] Kiyota Y, Schneeweiss S, Glynn RJ, et al. Accuracy of Medicare claims-based diagnosis of acute myocardial infarction: estimating positive predictive value on the basis of review of hospital records. Am Heart J. 2004; 148: 99-104. PMid: 15215798. https ://doi.org/10.1016/j.ahj.2004.02.013

[11] Aronsky D, Haug P, Lagor C, et al. Accuracy of administrative data for identifying patients with pneumonia. Am J Med Qual. 2005; 20 :
319-328. PMid: 16280395. https://doi.org/10.1177/106286 0605280358

[12] Gedeborg R, Furebring M, Michaëlsson K. Diagnosis-dependent misclassification of infections using administrative data variably affected incidence and mortality estimates in ICU patients. J Clin Epidemiol 2007; 60: 155-162. PMid: 17208121. https://doi.org/10.101 6/j.jclinepi.2006.05.013

[13] Sjoding MW, Iwashyna TJ, Dimick JB, et al. Gaming hospital-level pneumonia 30-day mortality and readmission measures by legitimate changes to diagnostic coding. Crit Care Med. 2015; 43: 989995. PMid: 25746747. https ://doi.org/10.1097/CCM. 000000 0000000862

[14] Rothberg MB, Pekow PS, Priya A, et al. Variation in diagnostic coding of patients with pneumonia and its association with hospital risk-standardized mortality rates: a cross-sectional analysis. Ann Intern Med. 2014; 160: 380-388. PMid: 24723078. https: //doi.org/10.7326/M13-1419

[15] Lindenauer PK, Lagu T, Shieh M, et al. Association of diagnostic coding with trends in hospitalizations and mortality of patients with pneumonia, 2003-2009. JAMA. 2012; 307: 1405-1413. PMid: 22474204. https://doi.org/10.1001/jama.2012.384

[16] Krumholz H, Normand S, Keenan P, et al. Hospital 30-day pneumonia readmission measure, methodology. Prepared by Yale New Haven Health Services Corporation/Center for Outcomes Research \& Evaluation for the Centers for Medicare \& Medicaid Services. Baltimore, MD: CMS; 2008.

[17] Yale New Haven Health Services Corporation/Center for Outcomes Research and Evaluation (YNHHSC/CORE). 2013 Measures Updates and Specifications: Acute Myocardial Infarction, Heart Failure, and Pneumonia 30-Day Risk-Standardized Mortality Measures (Version 7.0). Updated March 2013. Available from: http://sepas.srhs.com/wp-content/uploads/2014/ 01/Mort-AMI-HF-PN_MeasUpdtRept_v7.pdf

[18] Zuckerman RB, Sheingold SH, Orav EJ, et al. Readmissions, observation, and the Hospital Readmissions Reduction Program. N Engl J Med. 2016; 374: 1543-1551. PMid: 26910198. https: //doi.org/10.1056/NEJMsa1513024

[19] Gerhardt G, Yemane A, Hickman P, et al. Medicare readmission rates showed meaningful decline in 2012. Medicare Medicaid Res Rev. 2013; 3. https://doi.org/10.5600/mmrr.003.02.b01

[20] Carey K, Lin MY. Readmissions to New York hospitals fell for three target conditions from 2008 to 2012, consistent with Medicare goals. Health Aff (Millwood). 2015; 34: 978-985. PMid: 26056203 https://doi.org/10.1377/hlthaff .2014.1408

[21] Goldman LE, Chu PW, Osmond D, et al. The accuracy of present-onadmission reporting in administrative data. Health Serv Res. 2011; 46: 1946-1962. PMid: 22092023. https ://doi.org/10.1111/j . $1475-6773.2011 .01300 . x$ 